\title{
Erythroid progenitors differentiate and mature in response to endogenous erythropoietin
}

\author{
Takeshi Sato, ${ }^{1}$ Taira Maekawa, ${ }^{2}$ Sumiko Watanabe, ${ }^{3}$ Kohichiro Tsuji, ${ }^{1}$ \\ and Tatsutoshi Nakahata ${ }^{1}$ \\ ${ }^{1}$ Department of Clinical Oncology, \\ ${ }^{2}$ Department of Transfusion Medicine and Cell Therapy, and \\ ${ }^{3}$ Department of Molecular and Developmental Biology, The Institute of Medical Science, \\ The University of Tokyo, Tokyo, Japan \\ Address correspondence to: Taira Maekawa, Department of Transfusion Medicine and Cell Therapy, \\ The Institute of Medical Science, The University of Tokyo, 4-6-1 Shirokanedai, Minato-ku, Tokyo 108-8639, Japan. \\ Phone: 81-3-5449-5695; Fax: 81-3-5449-5438; E-mail: maekawa@ims.u-tokyo.ac.jp. \\ Tatsutoshi Nakahata's present address is: Department of Pediatrics, Graduate School of Medicine, Kyoto University, Kyoto, Japan. \\ Received for publication January 11, 2000, and accepted in revised form June 5, 2000.
}

\begin{abstract}
We reported previously that stimulation of glycoprotein 130 (gp130) by a combination of human IL6 and soluble IL- 6 receptor (sIL-6R) could support proliferation, differentiation, and terminal maturation of erythroid cells in the absence of erythropoietin (EPO) from human CD34 $4^{+}$cells in culture with stem cell factor (SCF). This observation suggested that differentiation of hematopoietic stem/progenitor cells to erythroid cells progressed according to an intrinsic program and that EPO receptor (EPOR) could be replaced by other cytokine receptors. In other words, EPOR appeared to be dispensable for erythropoiesis. Here we examined the role of EPOR in erythropoiesis stimulated by SCF, sIL-6R, and IL-6. Surprisingly, reduction of EPOR expression using antisense oligodeoxynucleotides suppressed erythropoiesis stimulated not only by SCF and EPO, but also by SCF, sIL-6R, and IL-6. EPO mRNA was detected in erythroid cells but not myeloid cells cultured in the presence of SCF, sIL-6R, and IL-6. Furthermore, high concentrations of anti-EPO-neutralizing antibody abrogated erythropoiesis in cultures without exogenous EPO. Based on these results, we suggest that erythroid progenitors themselves secrete EPO and that they have the potential to differentiate and mature in response to this endogenous EPO.
\end{abstract}

J. Clin. Invest. 106:263-270 (2000).

\section{Introduction}

Proliferation and differentiation of hematopoietic stem/progenitor cells are modulated by lineage-nonspecific early-acting and lineage-specific late-acting cytokines: e.g., stem cell factor (SCF) and IL-3 belong to the former, and erythropoietin (EPO), thrombopoietin (TPO), and G-CSF belong to the latter group (1). A number of studies have indicated that hematopoietic stem cells require both groups of cytokines to differentiate and fully mature into a specific lineage in vitro. With respect to erythropoiesis, a combination of one of the early-acting cytokines and EPO is essential for proliferation and differentiation of erythroid progenitors (2). The pivotal role of SCF in erythroid development was demonstrated by the severe macrocytic anemia in $\mathrm{Sl} / \mathrm{Sl}^{d}$ and $W / W^{v}$ mice mutated at the loci encoding SCF and its receptor c-kit, respectively $(3,4)$. The lineage-specific cytokine EPO is the crucial growth factor for erythropoiesis $(5,6)$. EPO acts by binding to its cognate receptor (EPOR), which is a member of the cytokine-receptor superfamily $(7,8)$ and is expressed on the surface of erythroid progenitors. Gene-targeting studies have indicated that EPO and EPOR are indispensable for the proliferation and survival of mature erythroid progenitors and their irreversible terminal differentiation $(9,10)$.
The IL- 6 receptor (IL-6R) system consists of a ligandbinding $\alpha$-subunit (IL-6R $\alpha$ ) and a signal-transducing $\beta$ subunit, glycoprotein 130 (gp130), which is commonly used by receptor complexes for the cytokines of the IL- 6 family (11). We found that most $\mathrm{CD} 34^{+}$cells in cord blood (CB) expressed gp130, but only 30-50\% expressed IL-6R $\alpha$, and that most erythroid, megakaryocytic, and immature hematopoietic progenitors were included in the CD34 IL$6 \mathrm{R} \alpha^{-}$population (12). Taga and Kishimoto found that a combination of soluble IL-6 receptor and IL-6 (sIL-6R/IL6) could activate gp130 and transduce the signal even in

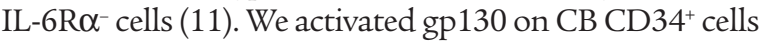
using sIL-6R/IL-6 and found that in the presence of SCF, erythropoiesis could be completed in the absence of exogenous EPO (13). These observations suggested that EPOR signaling may not be obligatory for erythropoiesis in vitro. Since Wu et al. demonstrated that SCF rapidly induced tyrosine phosphorylation of EPOR (14), one can speculate that EPOR may play a crucial role in transduction of erythroid differentiation signals without EPO. In other words, EPOR may function as an adapter molecule in erythroid cells even without binding its ligand EPO.

Here we have examined the role of EPOR in human erythropoiesis in the presence of SCF and sIL-6R/IL-6 by elimination of EPOR using antisense oligodeoxynu- 
cleotides (AS ODN) and by neutralization of EPO using an anti-EPO mAb. Surprisingly, we found that erythroid cells themselves produced EPO and that they stimulated their own erythroid differentiation in an autocrine manner. Erythroid progenitors therefore appear to have the potential to differentiate and to mature in response to endogenous EPO.

\section{Methods}

Cytokines and antibodies. Recombinant human (rh) IL6 and sIL-6R were obtained from Tosoh Co. (Ayase, Kanagawa, Japan), rhEPO was from Kirin Brewery (Tokyo, Japan), and rhSCF was from Amgen Inc. (Thousand Oaks, California, USA). Cytokine concentrations in culture medium were $100 \mathrm{ng} / \mathrm{mL}$ of SCF, $200 \mathrm{ng} / \mathrm{mL}$ of IL- $6,1,200 \mathrm{ng} / \mathrm{mL}$ of sIL-6R, and 2 $\mathrm{U} / \mathrm{mL}$ of EPO. Mouse mAb's for human (h) CD13 conjugated with phycoerythrin (PE) and for h-glycophorin A (h-GPA) conjugated with FITC were from Becton Dickinson (San Jose, California, USA) and PharMingen (San Diego, California, USA), respectively. Rabbit anti-hEPO-neutralizing Ab (IgG K-5) was provided by Kirin Brewery (15).

Cell preparation and suspension culture. Human CB, collected according to guidelines of the Institute of Medical Science, the University of Tokyo, was obtained from normal full-term deliveries after informed consent. Mononuclear cells (MNC) were separated by Ficoll/Paque density-gradient centrifugation after depletion of phagocytes with silica (Immuno Biological Laboratories, Fujioka, Gunma, Japan). CD34+ cells were purified from MNC using Dynabeads M-450 CD34 and DETACHaBEAD CD34 (Dynal AS, Oslo, Norway) according to the manufacturer's instructions, and over $95 \%$ of the separated cells were confirmed to be CD34 positive by flow-cytometry analyses.

The purified CD $34^{+}$cells were cultured at an initial density of $10^{3} / \mathrm{mL}$ at $37^{\circ} \mathrm{C}$ in a humidified atmosphere flushed with $5 \% \mathrm{CO}_{2} / 5 \% \mathrm{O}_{2} / 90 \% \mathrm{~N}_{2}$. Culture mixtures contained $\alpha$-medium (Flow Laboratories, Rockville, Maryland, USA) supplemented with 20\% FCS (HyClone, Logan, Utah, USA), 1\% BSA fraction V (Sigma Chemical Co., St. Louis, Missouri, USA), and various combinations of cytokines. After 1 week, half of the culture mixture was exchanged with fresh mixture containing the same supplements. Serum-free culture contained components identical to those in the serum-containing culture except that $1 \%$ pure BSA (Sigma Chemical Co.), $10 \mu \mathrm{g} / \mathrm{mL}$ of h-recombinant insulin (Sigma Chemical Co.), $200 \mu \mathrm{g} / \mathrm{mL}$ of h-transferrin (Sigma Chemical Co.), $160 \mu \mathrm{g} / \mathrm{mL}$ of soybean lecithin (Sigma Chemical Co.), and $96 \mu \mathrm{g} / \mathrm{mL}$ of cholesterol (Nacalai Tesque, Kyoto, Japan) replaced BSA fraction $\mathrm{V}$ and FCS.

Oligodeoxynucleotides and antisense treatment. All ODNs were synthesized using a large-scale DNA synthesizer and purified by reverse-phase HPLC, followed by dialysis (Genset Inc., Paris, France). The ODNs used in this study were 18 nucleotides in length, and the last three nucleotides at both ends had their internucleotidic linkages phosphorothioated (PS). These chimeric ODNs with partial PS-modification, in which end capping was used to prevent nuclease hydrolysis, were used because of their significantly decreased propensity for nonspecific and nonantisense effects sometimes observed in studies using uniformly PS-modified ODNs (16). The concentrated stock solutions were kept at $4{ }^{\circ} \mathrm{C}$ until use. We designed AS ODN targeted toward the AUG initiation region of the hEPOR mRNA $(17,18)$. Sense $(S)$ and random (RAN) sequences were used as controls. The sequences of these ODNs were as follows (s and $\mathrm{N}$ indicate a phosphorothioate and either one of A, T, G, C, respectively): hEPOR/AS, 5'-CsGsCsCCCGAGGTGGTCsCsAsT-3'; hEPOR/S, 5'-AsTsGsGACCACCTCGGGsGsCsG-3'; hEPOR/RAN, 5'-NsNsNsNNNNNNNNNNNNsNsNsN-3'. To suppress the expression of $\mathrm{hEPOR}$, these ODNs were initially added to cultures at a concentration of $10 \mu \mathrm{M}$, 3 hours before addition of cytokines, and on day 7 of culture $5 \mu \mathrm{M}$ ODNs were added again after half of the medium was changed.

RT-PCR. Using the QuickPrep Micro mRNA Purification Kit (Amersham Pharmacia Biotech, Uppsala, Sweden), mRNAs were isolated from cells cultured under various conditions and cDNAs were synthesized from the same amount $(0.5 \mu \mathrm{g})$ of each mRNA using the T-primed First-Strand Kit (Amersham Pharmacia Biotech) according to the manufacturer's instructions. The sequences of PCR primers were as follows: hEPO sense, 5'-ATGTGGATAAAGCCGTCAGTG3'; hEPO antisense, 5'-GCAGTGATTGTTCGGAGTGG-3'; hEPOR sense, 5'-TGTATCATGGACCACCTCGG-3'; hEPOR antisense, 5'-ACCAAGTCCTCCAACCGCTC-3'; hGPA sense, 5'-ACAAATGATACGCACAAACGG-3'; hGPA antisense, 5'-GTGTCAGGTGAGGGGAGAGG-3'. The PCR primer sets for $\mathrm{h} \beta$-actin were purchased from CLONTECH, Inc. (Palo Alto, California, USA). All of these PCR primer sets were intron spanning, and we confirmed that they could discriminate between PCR products generated from cDNA and those generated from contaminating genomic DNA. The cDNAs were amplified with Taq polymerase (Ampli Taq Gold; Perkin-Elmer Applied Biosystems, Foster City, California, USA) using a GenAmp PCR system 2400 thermal cycler (Perkin-Elmer). Each PCR cycle consisted of denaturation at $94^{\circ} \mathrm{C}$ for 30 seconds, annealing for 30 seconds, and extension at $72^{\circ} \mathrm{C}$ for 30 seconds. The annealing temperatures for $\mathrm{h} E P O, \mathrm{~h} E P O R$, $\mathrm{hGPA}$, and $\mathrm{h} \beta$-actin were $55.0^{\circ} \mathrm{C}, 60.0^{\circ} \mathrm{C}, 53.5^{\circ} \mathrm{C}$, and $55.0^{\circ} \mathrm{C}$, respectively. PCR products were electrophoresed on $7.5 \%$ polyacrylamide or $2.0 \%$ agarose gels, and visualized by ethidium bromide staining. Sequencing of PCR products. PCR products were subcloned into pCR2.1 TA-cloning vector (Invitrogen, San Diego, California, USA), and sequences were obtained using a Big Dye Terminator Cycle Sequencing Ready Reaction (Perkin-Elmer) and an ABI Prism 310 genetic analyzer (Perkin-Elmer), according to the manufacturers' instructions. 
Dianisidine staining. Erythroid cells were stained using dianisidine (3, 3'-dimethoxy benzidine, Sigma Chemical Co.) as described elsewhere (19).

Cell sorting. Cells were washed in PBS containing 2\% FCS and stained with mouse mAb's against both hCD13 (PE conjugated) and hGPA (FITC conjugated) for 30 minutes at $4^{\circ} \mathrm{C}$. As a control, a portion of each cell preparation was also stained with mouse class-matched control IgGs conjugated with PE and FITC. The stained cells were then washed in PBS three times and fractionated with FACS Vantage (Becton Dickinson).

Clonal serum-free culture and single colony RT-PCR for $h E P O$ production. The purified $\mathrm{CD} 34^{+}$cells were incubated in serum-free methylcellulose culture as reported previously $(12,13)$. Briefly, $1 \mathrm{~mL}$ of culture mixture containing $50 \mathrm{CD} 34^{+}$cells, $\alpha$-medium, $0.9 \%$ methylcellulose (Shinetsu Chemical, Tokyo, Japan), pure BSA, human transferrin (Sigma Chemical Co.), soybean lecithin (Sigma Chemical Co.), cholesterol (Nacalai Tesque), 0.05 mM 2-mercaptoethanol (Sigma Chemical Co.), and SCF plus sIL-6R/IL-6 was plated in 35$\mathrm{mm}$ Lux standard nontissue culture dishes (Nunc, Roskilde, Denmark) and incubated at $37^{\circ} \mathrm{C}$ in a humidified atmosphere flushed with $5 \% \mathrm{CO}_{2} / 5 \%$ $\mathrm{O}_{2} / 90 \% \mathrm{~N}_{2}$. After 14 days of culture, individual colonies were identified in situ according to the criteria as reported previously $(12,13)$.

Total RNA was extracted from individual colonies as described elsewhere (20). Briefly, single colonies formed by pure erythroid burst forming units (BFU-E), macrophage CFUs (CFU-M), granulocyte/macrophage CFUs (CFU-GM), and granulocyte CFUs (CFU-G) were individually lifted with a fine Pasteur pipette, and then resuspended in $20 \mu \mathrm{L}$ double-distilled water treated with dimethyl pyrocarbonate. To eliminate contaminating DNA, samples were treated with DNase I (Boehringer Mannheim, Mannheim, Germany), and cDNAs were synthesized as described above. After cDNA synthesis, $10 \mu \mathrm{L}$ of the cDNA solution was used for the RT-PCR reaction. The primers for $\mathrm{hEPO}$ and $\mathrm{h} \beta$ actin and PCR conditions for the RT-PCR reaction were the same as above.

Statistical analysis. For statistical comparison in scoring the total cell numbers, erythroid cell numbers, and percentages of hemoglobin-staining positive cells, a $t$ test was applied.

\section{Results}

EPOR was expressed in cells stimulated by SCF and sIL6R/IL-6. The EPOR gene is one of a class of erythroidspecific genes, such as GPA and globin. We examined whether EPOR and GPA were expressed in cells cultured with SCF and sIL-6R/IL-6, or SCF and EPO. Poly-A ${ }^{+}$ RNAs were extracted from $\mathrm{CB} \mathrm{CD} 34^{+}$cells cultured for 14 days in the presence of SCF and IL- 6 with or without sIL-6R, or in the presence of SCF and EPO, and were used as templates for the cDNA synthesis. Expression of EPOR and GPA was examined by RT-PCR. These erythroid-specific genes were expressed not only in cells cultured with SCF plus EPO, but also in cells cultured with SCF plus sIL-6R/IL-6. No erythroid-specific genes were detected in cells cultured in the presence of SCF plus IL-6 without sIL-6R (Figure 1).

Effects of AS ODN on the proliferation and differentiation of erythroid cells. First, the effect of the AS ODN on the expression of hEPOR mRNA was evaluated by RT-PCR. Poly-A RNAs were extracted from CB CD $34^{+}$cells cultured for 14 days with or without ODNs in the presence of SCF and EPO. Expression of hEPOR mRNA was significantly (but not completely) suppressed by addition of the AS ODN, whereas addition of either the S ODN or the RAN ODN did not affect hEPOR mRNA expression at all (Figure 2). Second, we examined the effects of the ODNs on SCF- and EPO-stimulated proliferation and differentiation. As shown in Figure 3, 500 purified human $\mathrm{CD}^{+} 4^{+}$cells stimulated by SCF and EPO proliferated and gave rise to $18.2 \times 10^{5} \pm 1.1 \times 10^{5}$ cells (mean $\pm \mathrm{SD}, n=3$ ) after 14 days of culture. The number and percentage of cells positive for hemoglobin staining were $16.2 \times 10^{5} \pm 0.6 \times 10^{5}$ cells (mean $\pm \mathrm{SD}, n=3$ ) and $90.8 \%$ $\pm 1.4 \%$ (mean $\pm \mathrm{SD}, n=3$ ), respectively. Addition of AS ODN reduced the total cell number to $40.1 \%$, and the percentage and number of hemoglobin positive cells to $32.9 \%$ and $13.2 \%$ of the levels observed in the absence of AS ODN, respectively. Addition of S ODN slightly suppressed the proliferation without influencing differentiation. This may be caused by the presence of four contiguous guanosine residues in S ODN (21). There were significant differences between cultures containing AS ODN and those containing control (S and RAN) ODNs with respect to total cell numbers $(P<0.02$ and $P<0.01$, respectively), numbers of hemoglobinized cells $(P<0.01$ and $P<0.01$, respectively), and hemoglobin positivity $(P$

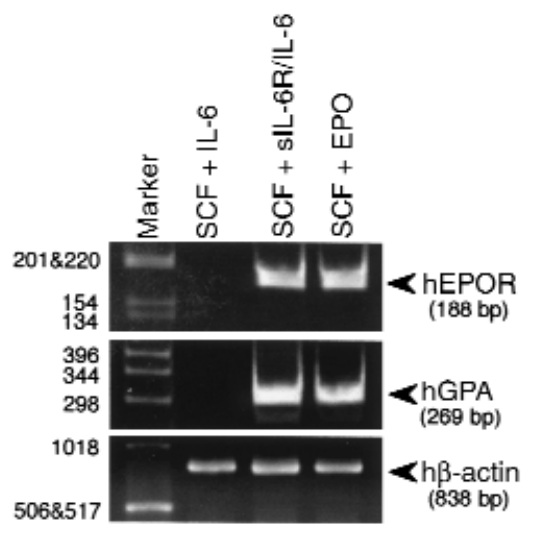

\section{Figure 1}

Expression of EPOR and erythroid-specific genes. CB CD34+ cells were cultured in the presence of SCF plus IL-6, with or without sIL$6 \mathrm{R}$, or in the presence of SCF plus EPO. After 14 days, the cells were harvested, and cDNAs were synthesized after mRNA extraction. EPOR and GPA mRNAs were detected by RT-PCR (33 cycles) in cells cultured both in the presence of SCF plus sIL-6R/IL- 6 and in the presence of SCF plus EPO, but not in cells cultured in the presence of SCF plus IL-6 without sIL-6R. The specificity of each PCR product was confirmed by sequencing. Size of the markers is indicated on the left. 


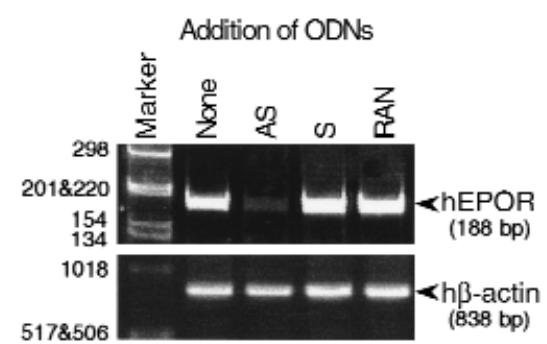

Figure 2

Effects of AS ODN on expression of EPOR. Five hundred CB CD34 cells were cultured in the presence of SCF and EPO, with or without ODNs. ODNs were initially added into cultures at $10 \mu \mathrm{M}$, and on day 7 of culture $5 \mu \mathrm{M}$ ODNs were added again after half the medium was changed. After 7 subsequent days of culture, the cells were harvested and cDNAs were synthesized after mRNA extraction. EPOR and $\beta$-actin mRNAs were detected using RT-PCR (33 cycles), and the specificity of the PCR products was confirmed by sequencing. Size of the markers is indicated on the left.

$<0.001$ and $P<0.001$, respectively) (Figure 3). Similar results were obtained in four separate experiments. These results suggest that the effects of EPO on the proliferation and differentiation of erythroid progenitors were suppressed by addition of AS ODN as a result of a reduced number of EPOR expression on the cell surface.

Reduced expression of EPOR suppressed erythropoiesis induced by SCF and sIL-6R/IL-6. We next examined the effects of reduction of EPOR expression by AS ODN on erythropoiesis stimulated by SCF and sIL-6R/IL-6. Addition of AS ODN resulted in significant suppression of both erythroid differentiation and proliferation, whereas addition of S ODN slightly suppressed the proliferation without affecting differentiation. RAN ODN had no effect on either proliferation or differentiation (Figure 4). There were significant differences between cultures containing AS ODN and those containing control (S and RAN) ODNs with respect to total cell numbers $(P<0.05$ and $P<0.02$, respectively), numbers of hemoglobinized cells $(P<0.01$ and $P<0.01$, respectively), and hemoglobin positivity $(P<0.001$ and $P<0.001$, respectively) (Figure 4). These findings are in apparent agreement with the results shown in Figure 3. It is likely that the existence of EPOR on erythroid progenitors is crucial for erythropoiesis induced by SCF and sIL6R/IL-6, even in the absence of exogenous EPO.

Detection of EPO $m R N A$ in cells stimulated by SCF and sIL$6 R / I L-6$. There are two possible explanations as to why the reduced expression of EPOR resulted in suppression of erythropoiesis stimulated by SCF and sIL-6R/IL-6 without addition of EPO. SCF may transduce signals for erythroid differentiation through EPOR, because cross-talk between c-kit and EPOR has been reported (14). Alternatively, SCF and sIL-6R/IL-6 may stimulate the production of endogenous EPO in the progeny of cultured CD34+ cells. Although the second hypothesis seemed unlikely in the human system because subsets of cells in the adult kidney and the fetal liver have been shown to be unique and specific populations that could produce EPO (22), we attempted to detect EPO mRNA using RT-PCR in cul- tured CD $34^{+}$cells stimulated by SCF and sIL-6R/IL-6. Poly-A RNA was extracted from $\mathrm{CB} \mathrm{CD} 34^{+}$cells cultured for 14 days in the presence of SCF and IL- 6 with or without sIL-6R and was used as template for cDNA synthesis. Because the PCR primers for $\mathrm{hEPO}$ are intron spanning, a RT-PCR product derived from cDNA can be differentiated from a product derived from contaminating genomic DNA on the basis of size (Figure 5a). A band corresponding to a 124-bp RT-PCR product was obtained only from the cDNA of cells cultured in the presence of SCF plus sIL-6R/IL-6, but not in cells cultivated with SCF plus IL-6 (Figure 5b). Sequencing analysis confirmed that this band was an amplicon from EPO mRNA, whose intron 4 had been spliced out. Both differentiation along an erythroid lineage and expression of EPO mRNA were observed in $\mathrm{CB} \mathrm{CD} 34^{+}$cells cultured in the presence of SCF plus sIL-6R/IL-6, whereas neither response was observed in cultures grown in the presence of SCF plus IL6 without sIL-6R. These results suggest that EPO mRNA expression in hematopoietic cells correlates with erythroid differentiation in the absence of exogenous EPO (Figure 1 and Figure 5b). Because EPO mRNA expression in murine macrophages (23) and murine bone marrow cells (24) has been reported, the possibility remained that a few contaminating cells producing EPO were present in the purified CD $34^{+}$fraction. However, this was found to be unlikely because EPO mRNA could not be detected in the $\mathrm{CD} 34^{+}$fraction similarly cultured in the presence of SCF plus IL-6 (Figure 5b).

Cells of erythroid lineage express EPO mRNA. Human CB $\mathrm{CD} 34^{+}$cells can differentiate into various lineages in the presence of SCF and sIL-6R/IL-6 (25). We therefore asked which cell lineage expressed EPO mRNA. CB $\mathrm{CD}^{+} 4^{+}$cells were cultured for 14 days in the presence of SCF and sIL-6R/IL-6, and then were double-stained with anti-hCD13-PE and anti-hGPA-FITC mAb's that recognize myeloid and erythroid cells, respectively.

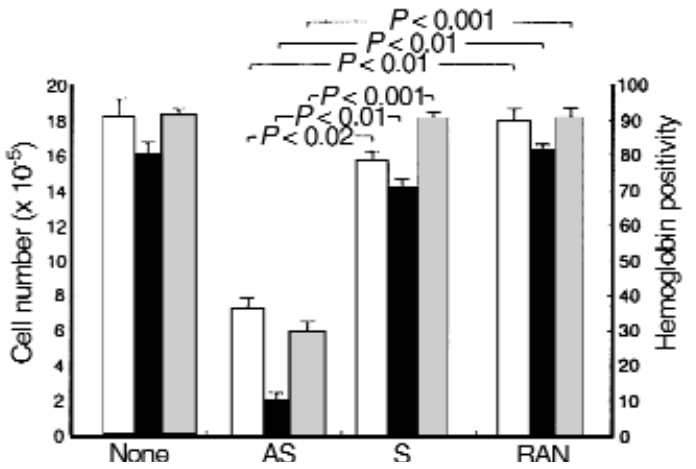

Figure 3

Effects of EPOR AS ODN on erythropoiesis in the presence of EPO. Five hundred $\mathrm{CB} \mathrm{CD} 34^{+}$cells were cultured for 14 days in the presence of SCF and EPO, with or without ODNs. Total cell numbers (open bars) were counted, and hemoglobin positivity (gray bars) was estimated after dianisidine staining. Numbers of hemoglobinized cells (black bars) were calculated from total cell numbers and hemoglobin positivity. Data are represented as the mean \pm SD of triplicate cultures. Similar results were obtained in four separate experiments. 


\section{Figure 4}

Effects of EPOR AS ODN on erythropoiesis induced by SCF and sIL6R/IL-6. Five hundred CB CD34+ cells were cultured for 14 days in the presence of SCF and sIL-6R/IL-6, with or without ODNs. Total cell numbers (open bars) were counted and hemoglobin positivity (gray bars) was estimated after dianisidine staining. Numbers of hemoglobinized cells (black bars) were calculated from total cell numbers and hemoglobin positivity. Data are represented as the mean \pm SD of triplicate cultures. Similar results were obtained in four separate experiments.
Stained cells were FACS sorted into CD13-GPA', $\mathrm{CD}_{13}^{+} \mathrm{GPA}^{-}$, and $\mathrm{CD}^{-}{ }^{-} \mathrm{GPA}^{-}$cells (Figure 6a), and each cell fraction was examined for EPO mRNA by RTPCR. EPO mRNA was detected in both the double-negative fraction and the $\mathrm{CD}_{13} \mathrm{GPA}^{+}$erythroid fraction, but not in the $\mathrm{CD} 13^{+} \mathrm{GPA}^{-}$myeloid fraction (Figure $6 \mathrm{~b})$. We then searched for trace amounts of EPO mRNA in the $\mathrm{CD} 13^{+} \mathrm{GPA}^{-}$fraction using nested PCR, but there was no evidence of EPO mRNA expression (data not shown). Therefore, EPO mRNA appeared to be expressed in erythroid cells but not in myeloid cells.

To further refine the findings, EPO mRNA expression of individual colonies was examined. We could detect the EPO mRNA expression in single colonies formed by pure BFU-E stimulated by SCF and sIL-6R/IL-6, but not in single colonies formed by pure CFU-M, pure CFUGM, and pure CFU-G (Table 1 and Figure 7). Similar results were obtained in five separate experiments.

Anti-EPO neutralizing Ab inbibited erythropoiesis stimulated by SCF and sIL-6R/IL-6. Preliminarily, we have tried to inhibit the expression of EPO by AS ODN targeting of several EPO sequences. However, we could not find the appropriate target-sequence for EPO. Therefore, we have tried to suppress the expression of EPO using anti-EPO neutralizing $\mathrm{Ab}$ as described previously $(26,27)$.

It has been reported that very high concentrations of anti-EPO-neutralizing Ab were required every 2 to 4 days to inhibit proliferation by autocrine EPO (26). Since we reported that addition of a moderate concentration of anti-hEPO-neutralizing Ab on day 0 failed to inhibit erythropoiesis stimulated by SCF and sIL-6R/IL-6 (13), we used higher concentrations of anti-hEPO-neutralizing $\mathrm{Ab}$ in order to reevaluate our previous results: that is, we added $0,0.5,5.0$, or $50.0 \mu \mathrm{g} / \mathrm{mL}$ of anti-hEPO-neutralizing $\mathrm{Ab}$ on days 0 and 7 of culture. Rabbit IgG was used as a control. Although $0.5 \mu \mathrm{g} / \mathrm{mL}$ of anti-hEPO-neutralizing $\mathrm{Ab}$ significantly suppressed erythropoiesis in the presence of EPO and SCF, the erythropoiesis stimulated by SCF and sIL-6R/IL-6 was not affected at all. However, 50 $\mu \mathrm{g} / \mathrm{mL}$ of anti-hEPO-neutralizing Ab completely inhibited erythropoiesis stimulated by SCF and sIL-6R/IL-6 (Figure 8). To exclude the possibility that bovine EPO contained in FCS had stimulated erythroid differentiation through hEPOR in the absence of hEPO, we performed serum-free cultures. Results similar to those found in serum-containing cultures were obtained (Table 2).

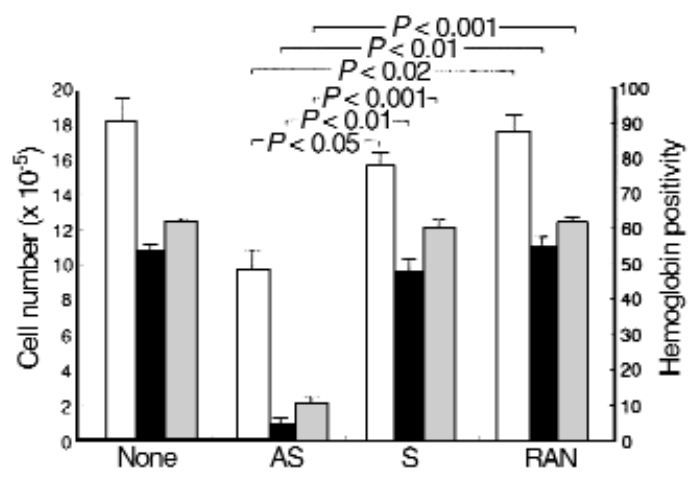

\section{Discussion}

Erythroid progenitors express EPOR on their cell surface and proliferate, differentiate, and mature in the presence of EPO (28). There are two hypotheses for EPOR signaling. First, EPOR generates erythroid-specific signals that lead to erythroid differentiation. Second, EPOR signaling merely keeps erythroid progenitors alive and supports terminal maturation according to an intrinsic erythroid program. In other words, EPO does not directly affect erythroid differentiation, and EPOR can be replaced by other cytokine receptors capable of eliciting the proliferation and survival of ery-

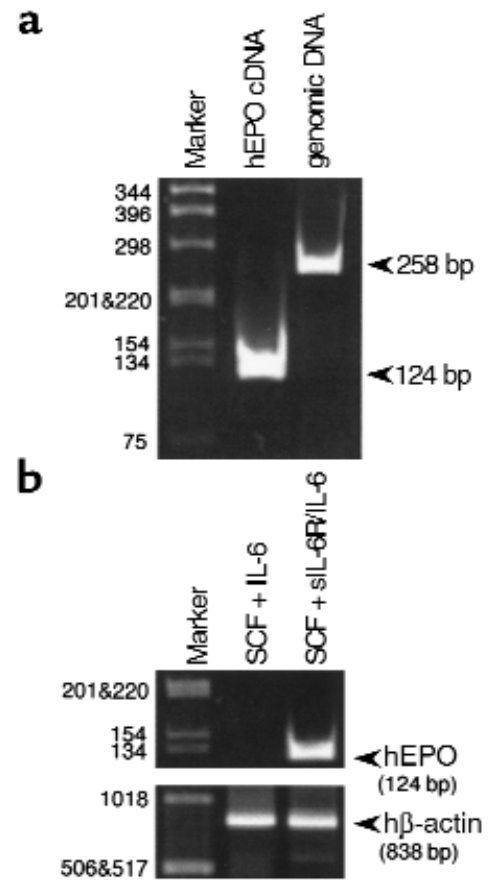

Figure 5

Detection of EPO mRNA in cells stimulated by SCF and sIL-6R/IL-6. (a) Human EPO cDNA and genomic DNA were amplified by PCR (33 cycles), and the PCR products were electrophoresed on a $7.5 \%$ polyacrylamide gel. The PCR products from hEPO CDNA and h-genomic DNA were $124 \mathrm{bp}$ and $258 \mathrm{bp}$, respectively. Sizes of the markers are indicated on the left. (b) $\mathrm{CB} C \mathrm{CD} 34^{+}$cells were cultured in the presence of SCF and IL-6, with or without sIL-6R. After 14 days, the cells were harvested and cDNAs were synthesized after mRNA extraction. EPO mRNA was detected only in cells cultured with SCF and sIL-6R/IL-6 after 40 cycles of RT-PCR, and the sequences were confirmed. 


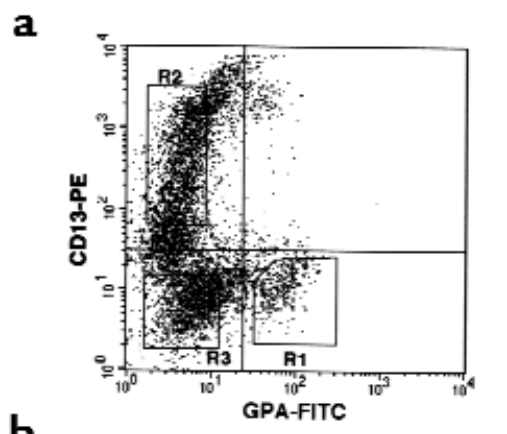

b

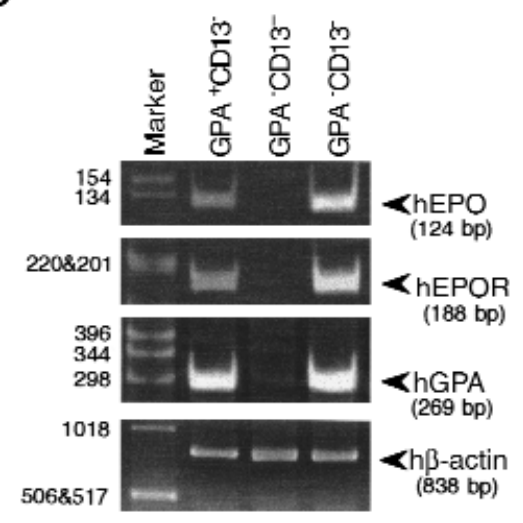

\section{Figure 6}

Cells of erythroid lineage express EPO mRNA. (a) CB CD34+ cells were cultured in the presence of SCF and sIL-6R/IL-6 for 14 days and the cells were FACS sorted into erythroids (R1), myeloids (R2), and other cells (R3) using anti-hGPA-FITC and anti-hCD13-PE mAb's. (b) cDNAs were synthesized from sorted cells after RNA extraction, and EPO mRNA detection was carried out using RT-PCR ( 40 cycles). Expression of EPOR, GPA, and $\beta$-actin mRNAs was also examined by RT-PCR (33 cycles). Size of the markers is indicated on the left.

throid progenitors. Previously, we presented evidence favorable to the latter hypothesis. We found that $\mathrm{CD} 34^{+}$ cells could differentiate into erythroid cells through the simultaneous activation of c-kit and gp130 in the absence of EPO (13). It is likely that c-kit and gp130 provided erythroid progenitors with sufficient signals for their proliferation and terminal differentiation even in the absence of EPO. Therefore, we expected that the elimination of EPOR expression by AS ODN would not affect erythropoiesis stimulated by SCF and sIL-6R/IL6. Contrary to our expectations, however, we found that

Table 1

EPO mRNA expression on single colonies stimulated by SCF plus sIL6R/IL-6 in serum-free culture

\begin{tabular}{lccc}
\hline $\begin{array}{l}\text { Type of } \\
\text { colonies }\end{array}$ & $\begin{array}{c}\text { No. of colonies } \\
\text { examined }\end{array}$ & $(+)$ & $(-)$ \\
BFU-E & 38 & 38 & 0 \\
CFU-M & 21 & 0 & 21 \\
CFU-GM & 24 & 0 & 24 \\
CFU-G & 8 & 0 & 8 \\
\hline
\end{tabular}

Fifty $\mathrm{CB} \mathrm{CD} 34^{+}$cells were cultured in $1 \mathrm{~mL}$ of serum-free methylcellulose media in the presence of SCF and sIL-6R/IL-6. RT-PCR analyses for EPO mRNA expression were performed for individual colonies lifted from culture dishes as described in Methods. the AS ODN-reduced expression of EPOR resulted in suppression of erythropoiesis stimulated not only by SCF plus EPO, but also by SCF plus sIL-6R/IL-6. Since the possibility of cross-talk between c-kit and EPOR in the murine cell line has been reported (14), one can speculate that erythroid differentiation signals may be transduced through EPOR in the absence of EPO. Sui et al. demonstrated that although SCF could induce phosphorylation of EPOR in human erythroid progenitors, it is not sufficient to transduce signals through EPOR and support cell growth, suggesting that SCF and EPO may activate MAP kinase through different pathways (29). Jacobs-Helber et al. also reported that phosphorylation of the EPOR by SCF does not initiate an EPO-like intracellular signal (30). In this study, we found that EPO mRNA was expressed in erythroid cells and that high concentrations of anti-EPO-neutralizing Ab completely abrogated erythropoiesis stimulated by SCF and sIL-6R/IL-6. These results suggest that human erythroid progenitors secrete EPO to induce their own differentiation.

Next, we determined which lineage of cells produced EPO. CD $34^{+}$cells cultured for 14 days in the presence of SCF and sIL-6R/IL-6 were FACS sorted into erythroids, myeloids, and other cell types using mAb's against erythroid-specific antigen GPA and myeloidspecific antigen CD13. EPO and EPOR mRNAs could be detected in the GPA-positive erythroid fraction, but not in the CD13-positive fraction, even using the nested PCR method. GPA, EPO, and EPOR mRNAs were also detected in the double-negative fraction (Figure $6 \mathrm{~b})$, which suggests that this fraction may contain erythroid progenitors including CFU erythroid (CFU-E) cells that express the GPA gene (31). These cells seem to express GPA on protein level in amounts insufficient to detect by immunological techniques.

Because EPO mRNA expression in murine macrophages has been reported (23), the possibility exists that accessory cells other than erythroid progenitors produce EPO. We observed that $\mathrm{CD}^{2} 4^{+}$cells stimulated by SCF and sIL-6R/IL-6 expressed EPO mRNA, whereas those stimulated by SCF and IL- 6 did not (Figure 5b). Furthermore, we observed that single BFU-E-derived colonies expressed EPO mRNA, whereas CFU-M-, CFU-GM-, and CFU-G-derived colonies did not (Table 1 and Figure 7).

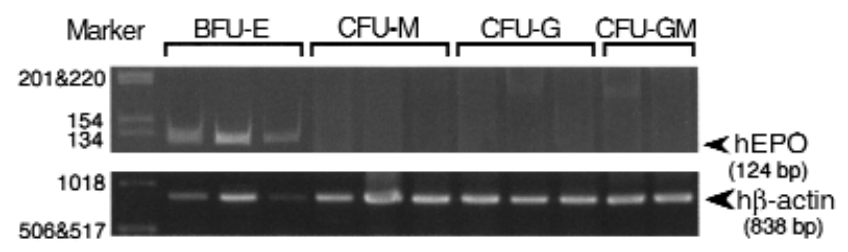

Figure 7

EPO mRNA expression on single colonies in clonal culture. Total RNA was extracted from single colonies formed by pure BFU-E, CFU-M, CFU$\mathrm{GM}$, and CFU-G. cDNAs were synthesized and EPO mRNA detection was carried out using RT-PCR (45 cycles). Examples of electrophoresis by RT-PCR aliquots from single colonies in an experiment are shown. Size of the markers is indicated on the left. 


\section{Figure 8}

Anti-hEPO neutralizing Ab abrogated erythropoiesis induced by SCF and sIL-6R/IL-6. CB CD34+ cells were cultured for 14 days in the presence of SCF plus EPO (open circles), or SCF plus sIL-6R/IL6 (filled circles), with $0,0.5,5.0$, or $50.0 \mu \mathrm{g} / \mathrm{mL}$ of anti-hEPOneutralizing $A b$. The same concentrations of rabbit IgG were added to control samples. After 7 days, when half of the medium was changed, the same concentration of anti-hEPO Ab or IgG was added. The number of hemoglobin-positive cells was calculated from total cell number and hemoglobin positivity determined by dianisidine staining. Data represent the percentage of hemoglobin-positive cells in each well with added anti-hEPO $\mathrm{mAb}$ relative to the corresponding control samples. Similar results were obtained in three separate experiments. produced in cells that had the potential to differentiate into an erythroid lineage, and findings in the present study are not due to the contamination of macrophages.

It seems that gp130 signaling in cells with the potential to differentiate into erythroid cells is crucial in vitro for erythropoiesis without EPO. However, its importance for erythropoiesis in vivo has not been clarified. Since the gp130-deficient mice embryo showed anemia due to impaired maturation of erythroid cells (32) and both the number of erythrocytes and hemoglobin values increased in sIL-6R/IL-6 double-transgenic mice (33), gp130 signaling may play a certain role in erythropoiesis in vivo. Because forced activation of gp130 in sIL-6R/IL-6 double-transgenic mice may induce endogenous EPO and enhance erythropoiesis and other lineages of blood cells also increased, it is suggested that gp130 signaling regulates both stem/multipotential and committed progenitors (34).

Goldwasser et al. suggested that murine multipotent progenitors, but not committed erythroid progenitors, possessed intracellular EPO autocrine activity (35). They proposed that commitment of multipotent progenitors was regulated by intracellular autocrine activity of cytokines. It also has been reported that $\mathrm{COOH}$-terminal-modified IL-3 is retained intracellularly where it stimulates the autocrine growth of $32 \mathrm{D}$ cells (36). Villeval et al. reported that autocrine stimulation by EPO required its secretion by UT-7 cells, which were engineered to produce EPO (26). They observed that high concentrations of anti-EPO-neutralizing $\mathrm{Ab}$ were required to inhibit proliferation induced by endogenous EPO. For example, an $\mathrm{Ab}$ dilution inhibiting approximately $100 \mathrm{U} / \mathrm{mL}$ of exogenous EPO was necessary to completely block the proliferation of the cells that secreted only 1 or $2 \mathrm{U}$ of endogenous EPO every 2 to 4 days, and they suggested that the autocrine stimulation induced by EPO occurred on the cell surface. It has been reported that some erythroleukemic cells producing EPO proliferate by an autocrine manner $(27,37,38)$. Mitjavila et al. reported that $60 \mu \mathrm{g} / \mathrm{mL}$ of anti-hEPO-neutralizing Ab inhibited $87 \%$ of spontaneous growth of EPO-producing leukemic cells

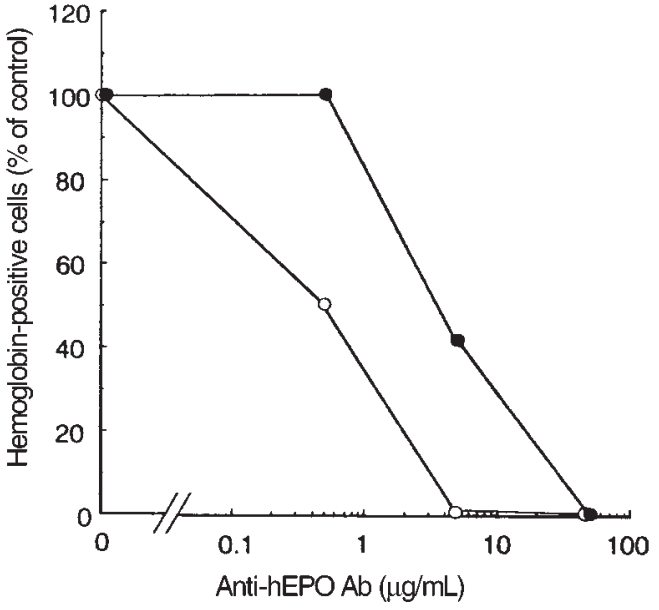

(37). Therefore it seems likely that high concentrations of anti-hEPO Ab are required to neutralize autocrine of EPO. Since we reported previously that addition of moderate concentration of anti-hEPO-neutralizing $\mathrm{Ab}$ on day 0 failed to inhibit erythropoiesis stimulated by SCF and sIL6R/IL-6 (13), we used higher concentrations of anti-hEPO-neutralizing $\mathrm{Ab}$ on days 0 and 7 of culture in the present study. Since $50 \mu \mathrm{g} / \mathrm{mL}$ of anti-hEPO-neutralizing $\mathrm{Ab}$ completely inhibited erythropoiesis in the absence of exogenous EPO, it is likely that erythropoiesis stimulated by SCF and sIL-6R/IL- 6 was due to endogenous EPO produced in the erythroid progenitors themselves. We also observed that erythropoiesis stimulated by SCF and sIL-6R/IL-6 was independent of cell density, and that the culture supernatant of erythroid cells grown in the presence of SCF and sIL-6R/IL-6 did not stimulate proliferation of hEPOR-transfected $\mathrm{Ba} / \mathrm{F} 3$ cells (data not shown). These results suggest that little EPO was secreted into the culture medium. In our previous study, anti-EPO-neutralizing $\mathrm{Ab}$ was added only on day 0 (13). Since anti-EPO-neutralizing $\mathrm{Ab}$ seemed to form immune complexes with exogenous EPO in culture media immediately after its addition, erythropoiesis in the presence of SCF and EPO could be suppressed by small amounts of it. When erythroid cells generated in the presence of SCF and sIL-6R/IL-6 gradually produce endogenous EPO along their differentiation during 2 weeks of culture, it is possible that anti-EPO-neutralizing $\mathrm{Ab}$ added on day 0 has lost its activity during the first 7 days of culture, and supple-

\section{Table 2}

Effects of anti-EPO Ab on erythropoiesis stimulated by SCF plus sIL$6 \mathrm{R} / \mathrm{IL}-6$ in serum-free culture

$\begin{array}{cc}\text { Anti-hEPO Ab }(\mu \mathrm{g} / \mathrm{mL}) & \text { Hemoglobin-positive cells (\% of control) } \\ - & 100 \\ 5.0 & 49 \pm 8 \\ 50.0 & 0 \pm 0\end{array}$

$\mathrm{CB} C D 34^{+}$cells were cultured in serum-free media for 14 days in the presence of SCF plus sIL-6R/IL-6, with $0,5.0$, or $50.0 \mu \mathrm{g} / \mathrm{mL}$ of anti-hEPO-neutralizing $A b$. Data are presented as mean $\pm S D$ in triplicate cultures. Similar results were obtained in three separate experiments. 
ments on day 7 are required. Taken together, we propose that EPO is secreted from mature erythroid progenitors and stimulates their own differentiation in the presence of SCF and sIL-6R/IL-6 in an autocrine manner. Complete abrogation of erythropoiesis by $50 \mu \mathrm{g} / \mathrm{mL}$ of anti$\mathrm{EPO} \mathrm{Ab}$ suggests that cross-talk between c-kit and EPOR may not contribute to erythroid differentiation induced by SCF and sIL-6R/IL-6.

However, it has been reported that a combination of SCF and TPO rescues erythroid colony formation from mouse embryos lacking the EPOR (39). Socolovsky et al. also reported that the prolactin receptor rescues $E P O R^{-/-}$erythroid progenitors and replaces EPOR in a synergistic interaction with c-kit (40). These reports suggest existence of an EPO-independent pathway of erythropoiesis. Recently, it has been shown that endogenous EPO could be detected in erythroid colonies stimulated by an EPO-mimicking peptide (41). Our present study indicates that endogenous EPO production in mature erythroid progenitors forms a part of the intrinsic program of normal erythroid development. Further studies are required to clarify these problems.

\section{Acknowledgments}

We thank A. Kaneko for technical support and P. Hughes for comments on the manuscript. This work was supported in part by a Grant-in-Aid for Scientific Research from the Ministry of Education, Science, Sports and Culture of Japan to T. Maekawa (11670983, 12217039) and T. Nakahata (11357008).

1. Ogawa, M. 1993. Differentiation and proliferation of hematopoietic stem cells. Blood. 81:2844-2853.

2. Migliaccio, A.R., Vannucchi, A.M., and Migliaccio, G. 1996. Molecular control of erythroid differentiation. Int. J. Hematol. 64:1-29.

3. Zsebo, K.M., et al. 1990. Stem cell factor is encoded at the Sl locus of the mouse and is the ligand for the c-kit tyrosine kinase receptor. Cell. 63:213-224.

4. Ikuta, K., Ingolia, D.E., Friedman, J., Heimfeld, S., and Weissman, I.L. 1991. Mouse hematopoietic stem cells and the interaction of c-kit receptor and steel factor. Int. J. Cell Cloning. 9:451-460.

5. Krantz, S.B. 1991. Erythropoietin. Blood. 77:419-434.

6. Zhu, Y., and D'Andrea, A.D. 1994. The molecular physiology of erythropoietin and the erythropoietin receptor. Curr. Opin. Hematol. 1:113-118.

7. D'Andrea, A.D., Lodish, H.F., and Wong, G.G. 1989. Expression cloning of the murine erythropoietin receptor. Cell. 57:277-285.

8. Bazan, J.F. 1990. Structural design and molecular evolution of a cytokine receptor superfamily. Proc. Natl. Acad. Sci. USA. 87:6394-6398.

9. Wu, H., Liu, X., Jaenisch, R., and Lodish, H.F. 1995. Generation of committed erythroid BFU-E and CFU-E progenitors does not require erythropoietin or the erythropoietin receptor. Cell. 83:59-67.

10. Lin, C.S., Lim, S.K., D'Agati, V., and Costantini, F. 1996. Differential effects of an erythropoietin receptor gene disruption on primitive and definitive erythropoiesis. Genes Dev. 10:154-164.

11. Taga T., and Kishimoto T. 1997. Gp130 and the interleukin-6 family of cytokines. Annu. Rev. Immunol. 15:797-819.

12. Tajima, S., et al. 1996. Analysis of interleukin 6 receptor and gp130 expressions and proliferative capability of human CD34+ cells. J. Exp. Med. 184:1357-1364.

13. Sui, X., et al. 1996. Erythropoietin-independent erythrocyte production: signals through gp130 and c-kit dramatically promote erythropoiesis from human CD34+ cells. J. Exp. Med. 183:837-845.

14. Wu, H., Klingmuller, U., Besmer, P., and Lodish, H.F. 1995. Interaction of the erythropoietin and stem-cell-factor receptors. Nature. 377:242-246.
15. Nishi, N., et al. 1990. Induction of mixed erythroid-megakaryocyte colonies and bipotential blast cell colonies by recombinant human erythropoietin in serum-free culture. Blood. 76:1330-1335.

16. Uhlmann, E., Ryte, A., and Peyman, A. 1997. Studies on the mechanism of stabilization of partially phosphorothioated oligonucleotides against nucleolytic degradation. Antisense Nucleic Acid Drug Dev. 7:345-350.

17. Jones, S.S., D’Andrea, A.D., Haines, L.L., and Wong, G.G. 1990. Human erythropoietin receptor: cloning, expression, and biologic characterization. Blood. 76:31-35.

18. Noguchi, C.T., et al. 1991. Cloning of the human erythropoietin receptor gene. Blood. 78:2548-2556.

19. Guimaraes, J.E., Berney, J.J., Francis, G.E., and Hoffbrand, A.V. 1984. The identification of mixed granulocytic-erythrocytic colonies in vitro. Exp. Hematol. 12:535-538.

20. Curtis, D.J., Metcalf, D., Alexander, B., and Begley, C.G. 2000. Leukemic cells from murine myeloid leukemia display an intrinsic ability for autonomous proliferation. Exp. Hematol. 28:36-45.

21. Burgess, T.L., et al. 1995. The antiproliferative activity of c-myb and cmyc antisense oligonucleotides in smooth muscle cells is caused by a nonantisense mechanism. Proc. Natl. Acad. Sci. USA. 92:4051-4055.

22. Ebert, B.L., and Bunn, H.F. 1999. Regulation of the erythropoietin gene. Blood. 94:1864-1877.

23. Vogt, C., Pentz, S., and Rich, I.N. 1989. A role for the macrophage in normal hemopoiesis: III. In vitro and in vivo erythropoietin gene expression in macrophages detected by in situ hybridization. Exp. Hematol. 17:391-397.

24. Hermine, O., Beru, N., Pech, N., and Goldwasser, E. 1991. An autocrine role for erythropoietin in mouse hematopoietic cell differentiation [erratum 1992, 79:3397]. Blood. 78:2253-2260.

25. Sui, X., et al. 1995. gp130 and c-Kit signalings synergize for ex vivo expansion of human primitive hemopoietic progenitor cells. Proc. Natl. Acad. Sci. USA. 92:2859-2863.

26. Villeval, J.L., et al. 1994. Autocrine stimulation by erythropoietin (Epo) requires Epo secretion. Blood. 84:2649-2662.

27. Choppin, J., et al. 1984. Characterization of erythropoietin produced by IW32 murine erythroleukemia cells. Blood. 64:341-347.

28. Metcalf, D., and Nicola, N.A. 1995. The hemopoietic colony-stimulating factors. Cambridge University Press. Melbourne, Australia. 328 pp.

29. Sui, X., Krantz, S.B., You, M., and Zhao, Z. 1998. Synergistic activation of MAP kinase (ERK1/2) by erythropoietin and stem cell factor is essential for expanded erythropoiesis. Blood. 92:1142-1149.

30. Jacobs-Helber, S.M., Penta, K., Sun, Z., Lawson, A., and Sawyer, S.T. 1997. Distinct signaling from stem cell factor and erythropoietin in HCD57 cells. J. Biol. Chem. 272:6850-6853.

31. Okumura, N., Tsuji, K., and Nakahata, T. 1992. Changes in cell surface antigen expressions during proliferation and differentiation of human erythroid progenitors. Blood. 80:642-650.

32. Yoshida, K., et al. 1996. Targeted disruption of gp130, a common signal transducer for the interleukin 6 family of cytokines, leads to myocardial and hematological disorders. Proc. Natl. Acad. Sci. USA. 93:407-411.

33. Peters, M., et al. 1997. Extramedullary expansion of hematopoietic progenitor cells in interleukin (IL)-6-sIL-6R double transgenic mice. J. Exp. Med. 185:755-766.

34. Peters, M., Muller, A.M., and Rose-John, S. 1998. Interleukin-6 and soluble interleukin-6 receptor: direct stimulation of gp130 and hematopoiesis. Blood. 92:3495-3504.

35. Goldwasser, E., Hermine, O., Pech, N., and Stage-Marroquin, B. 1994. Internal autocrine regulation of the early stages of hemopoiesis. Ann. NY Acad. Sci. 718:326-330.

36. Dunbar, C.E., Browder, T.M., Abrams, J.S., and Nienhuis, A.W. 1989. $\mathrm{COOH}$-terminal-modified interleukin-3 is retained intracellularly and stimulates autocrine growth. Science. 245:1493-1496.

37. Mitjavila, M.T., et al. 1991. Autocrine stimulation by erythropoietin and autonomous growth of human erythroid leukemic cells in vitro. J. Clin. Invest. 88:789-797.

38. Stage-Marroquin, B., Pech, N., and Goldwasser, E. 1996. Internal autocrine regulation by erythropoietin of erythroleukemic cell proliferation. Exp. Hematol. 24:1322-1326.

39. Kieran, M.W., Perkins, A.C., Orkin, S.H., and Zon, L.I. 1996. Thrombopoietin rescues in vitro erythroid colony formation from mouse embryos lacking the erythropoietin receptor. Proc. Natl. Acad. Sci. USA. 93:9126-9131.

40. Socolovsky, M., Fallon, A.E., and Lodish, H.F. 1998. The prolactin receptor rescues EpoR-/- erythroid progenitors and replaces EpoR in a synergistic interaction with c-kit. Blood. 92:1491-1496.

41. Stopka, T., Zivny, J.H., Stopkova, P., Prchal, J.F., and Prchal, J.T. 1998. Human hematopoietic progenitors express erythropoietin. Blood. 91:3766-3772. 